\title{
Evaluation of Tumor Elasticity and Clinicopathologic Factors Affecting Neoadjuvant Chemotherapy Response in Patients with Breast Cancer
}

\author{
Young Ah Kim, M.D. ${ }^{1}$, Jeong Yeong Park, M.D., Ph.D. ${ }^{1}$, Jung Eun Choi, M.D., Ph.D. ${ }^{1}$, Su Hwan Kang, M.D., Ph.D. ${ }^{1}$, \\ Young Kyung Bae, M.D., Ph.D. ${ }^{2}$, Mi Soo Hwang, M.D., Ph.D. ${ }^{3}$, Soo Jung Lee, M.D., Ph.D. ${ }^{1}$ \\ ${ }^{1}$ Department of Surgery, ${ }^{2}$ Department of Pathology, ${ }^{3}$ Department of Radiology, Yeungnam University Medical Center, Daegu, Korea
}

Purpose: This study aimed to determine the clinicopathologic factors, including tumor elasticity, affecting neoadjuvant chemotherapy response in breast cancer. Methods: Among 95 patients who received neoadjuvant chemotherapy for clinical stage lla-lllc primary breast cancer, 75 underwent strain elastography assessments. The patients were divided into soft and hard tumor groups based on the Tsukuba elasticity scoring system. Pathologic factors, including tumor cellularity and stromal characteristics, were evaluated using core needle biopsy specimens collected before neoadjuvant chemotherapy. Pathologic complete response (pCR) was defined as the absence of invasive carcinoma in the breast and axillary lymph nodes. Residual cancer burden (RCB) was also calculated in 79 cases. Results: Twenty-two patients achieved pCR (23.2\%). The rates of estrogen receptor (ER) negativity ( $p=0.04)$, progesterone receptor (PR) negativity $(p=0.03)$, and nuclear grade $3(p=0.03)$ were higher in patients with $p C R$ than those in patients without $p C R$. The rates of PR negativity $(p=0.03)$, nuclear grade 3 $(p=0.01)$, and high tumor-infiltrating lymphocyte (TIL) levels $(p=0.04)$ were significantly higher in the favorable RCB group (RCB-0 and I) than those in the unfavorable RCB group (RCB-II and III). No significant difference in tumor elasticity was observed between the groups $(p=0.30)$. Hormone receptor (HR) negativity was an independent predictor of favorable RCB in the multivariate analysis $(p=0.04)$. Conclusion: Tumor elasticity was not associated with pCR or RCB. HR negativity was an independent predictor of favorable RCB.

Key Words: Breast neoplasms, Elasticity imaging techniques, Neoadjuvant therapy, Ultrasonography

\section{INTRODUCTION}

Neoadjuvant chemotherapy is commonly performed for breast cancer patients because of its benefits [1-3]. Accordingly, efforts to predict neoadjuvant chemotherapy responses have been reported. Biologic subtypes are well known for their predictive value for neoadjuvant chemotherapy response [4-7]. Some studies [8-10] reported a significant correlation between a higher tumor-infiltrating lymphocyte (TIL) density and pathologic complete response (pCR). Other studies have reported the predictive value of pathological factors such as Ki-67 index, p53, topoisomerase II alpha, and nuclear factor kappa/B-cell lymphoma-2 [11-14]. Despite these studies, no predictive model can accurately predict whether patients will achieve pCR after neoadjuvant chemotherapy.

As malignant tumors progress, the amounts of stromal tissues, es-

Correspondence: Soo Jung Lee, M.D., Ph.D.

Department of Surgery, Yeungnam University Medical Center, 170 Hyeonchungro, Nam-gu, Daegu 42415, Korea

Tel: +82-53-620-3580, Fax: +82-53-624-1213, E-mail: crystallee@med.yu.ac.kr

Received: Sep 7, 2020 Revised: Feb 15, 2021 Accepted:Mar 28, 2021 pecially the extracellular matrix, such as collagen, elastin, and fibrous adhesive protein, increase. These stromal tissues contribute to a poor response to neoadjuvant chemotherapy by acting as a physical barrier and increasing interstitial fluid pressure. Elevated interstitial fluid pressure interferes with the diffusion of chemotherapy agents and aggravates hypoxia, which degrades or deactivates chemotherapeutic agents by compressing intratumoral vessels [15]. Accumulation of the fibrous component of the stroma in the tumor results in decreased tumor elasticity that is categorized as a hard tumor in elastography. Chamming et al. [16] reported a correlation between tumor elasticity measured by shear wave elastography and fibrosis in a human breast cancer model. Thus, tumor elasticity measured by elastography might be a predictive factor for neoadjuvant chemotherapy response.

Elastography can be simultaneously performed during conventional ultrasonography (US), which is a basic imaging tool for breast cancer. Elastography is usually initially performed to differentiate between benign and malignant tumors $[17,18]$. Recently, several studies have assessed whether strain elastography or shear wave elastography can predict neoadjuvant chemotherapy response in patients with breast 
cancer [19-21].

The present study evaluated the effects of clinicopathologic factors, including tumor elasticity, on neoadjuvant chemotherapy response in patients with stage II or III breast cancer.

\section{METHODS}

From April 2014 to March 2017, 95 patients were scheduled to receive neoadjuvant chemotherapy for clinical stage IIa-IIIc breast cancer at Yeungnam University Hospital. Mammography, breast US, strain elastography, and positron emission tomography (PET) were performed for clinical staging. Fine needle aspiration or axillary surgery (sentinel lymph node biopsy [SLNB] or axillary lymph node dissection $[A L N D]$ ) was performed to assess lymph node (LN) metastasis if such metastasis was not obvious in imaging studies. The Institutional Review Board of Yeungnam University Hospital approved this prospective study and written informed consent was obtained from each patient (IRB No. 2014-01-459).

\section{US and strain elastography}

US and strain elastography were performed using a Philips iU22 ultrasound system (Philips Medical System, Bothell, USA) with a 5-12 $\mathrm{MHz}$ linear transducer by an expert radiologist with $>30$ years of experience in US and $>2$ years of experience in strain elastography. To perform strain elastography, light manual compression was performed above the targeted tumors and the surrounding normal tissues. Real-time elastography images were displayed on a 256-color map and tumor elasticity was evaluated using the Tsukuba elasticity scoring system (score 1-5) [22]. A cut-off score between 3 and 4 was used to categorize patients into soft (Tsukuba score $\leq 3$; Figure 1A) and hard (Tsukuba score $\geq 4$; Figure 1B) tumor groups [22]. Strain elastography was performed in 74 cases. In 47 cases, a second US was performed for follow-up after the first cycle of neoadjuvant chemotherapy. The reduction rate of tumor size between the first and second US was calculated.

\section{Neoadjuvant chemotherapy and operations}

Sixty-three patients received four cycles of AC (adriamycin $60 \mathrm{mg}$ / $\mathrm{m}^{2}$ and cyclophosphamide $600 \mathrm{mg} / \mathrm{m}^{2}$ ) followed by four cycles of paclitaxel (175 mg/m²), eight patients received four cycles of AC followed by four cycles of docetaxel $\left(75 \mathrm{mg} / \mathrm{m}^{2}\right), 16$ patients received eight cycles of docetaxel $\left(75 \mathrm{mg} / \mathrm{m}^{2}\right)$ plus adriamycin $\left(50 \mathrm{mg} / \mathrm{m}^{2}\right)$, and four patients received eight cycles of docetaxel $\left(75 \mathrm{mg} / \mathrm{m}^{2}\right)$ plus epirubicin $(50$ $\mathrm{mg} / \mathrm{m}^{2}$ ). The other four patients received eight cycles of FEC (5-FU $500 \mathrm{mg} / \mathrm{m}^{2}$, epirubicin $100 \mathrm{mg} / \mathrm{m}^{2}$, and cyclophosphamide $500 \mathrm{mg} /$ $\mathrm{m}^{2}$ ). In 22 cases with human epidermal growth factor receptor 2 (HER2)-positive breast cancers, trastuzumab was administered concurrently with taxane following four cycles of AC.
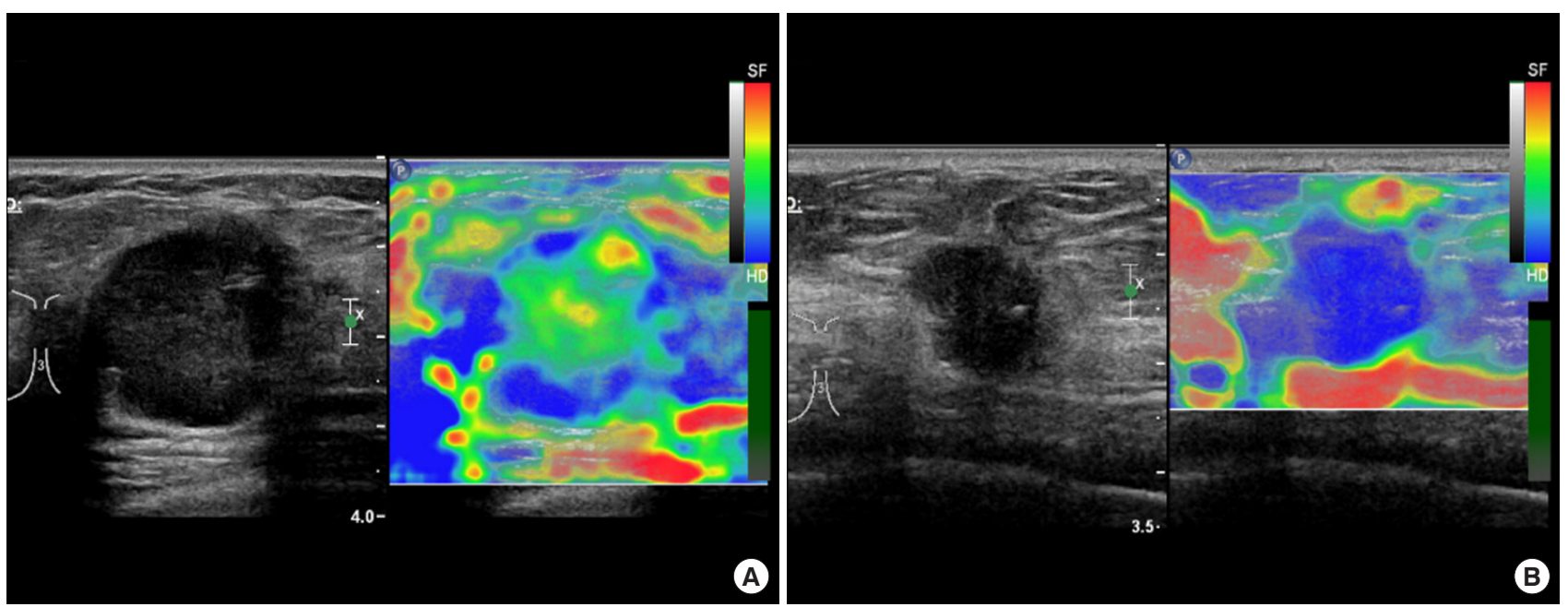

Figure 1. Conventional ultrasonography and strain elastography of soft (A) and hard (B) tumors. (A) A $3.3 \mathrm{~cm}$ heterogeneous hypoechoic mass is observed on conventional ultrasonography (left). On strain elastography (right), most of the tumor is green, with some blue areas. The Tsukuba elasticity score was 2. (B) A 2.3 $\mathrm{cm}$ irregular shape hypoechoic mass on conventional ultrasonography (left) and surrounding tissues are blue on strain elastography (right). The Tsukuba elasticity score was 5. 
After neoadjuvant chemotherapy, the patients underwent breast-conserving surgery (BCS), mastectomy, or skin-sparing mastectomy. In 45 patients who underwent SLNB or ALND before neoadjuvant chemotherapy, axillary surgery was omitted. ALND was performed in 13 patients. In the other 50 cases, SLNB was performed and ALND was added when tumor metastasis of sentinel LN was confirmed by frozen section biopsy.

\section{Evaluation of pathologic characteristics}

Histologic type; nuclear grade; TILs; tumor cellularity; stromal characteristics; estrogen receptor (ER), progesterone receptor (PR), and HER2 statuses; and Ki-67 index were evaluated from core needle biopsy $(\mathrm{CNB})$ specimens obtained before the administration of neoadjuvant chemotherapy. The evaluation of TIL was performed on hematoxylin and eosin H\&E-stained slides according to the International TIL Working Group recommendations [23]. The TIL density was assessed inside the borders of the invasive carcinoma and calculated by expressing stromal areas occupied by lymphocytes and plasma cells as percentages of total intratumoral stromal areas. Tumor cellularity was evaluated in H\&E-stained slides as the percentage of tumor area occupied by tumor cells. The stromal characteristics were assessed in H\&E-stained slides and categorized into three types (fibroblastic, collagenous, or myxoid) according to the dominant component. Immunohistochemistry (IHC) for ER (SP1, Ventana Medical Systems, Tucson, AZ, USA), PR (IE2, Ventana Medical Systems), and HER2 (4B5, Ventana Medical Systems) were performed. ER and PR positivity were defined as $>1 \%$ positive tumor cells. HER 2 positivity was indicated by an IHC score of $3+$ or HER2/CEP17 ratio of $\geq 2.0$, or an average HER2 copy number of $\geq 6.0$ in silver-enhanced in situ hybridization (SISH). After surgery of the breast and axillary LNs, histologic type, histologic grade, tumor size, LN tumor metastasis, TILs, tumor cellularity, ER, PR, HER2, and Ki-67 index were evaluated.

\section{Pathologic response to neoadjuvant chemotherapy}

pCR was defined as the absence of invasive carcinoma in the surgically resected breast (ypT0 and ypTis) and axillary LNs (ypN0). We divided the patients into $\mathrm{pCR}$ and non-pCR groups. The residual cancer burden (RCB) [24] was calculated using the MD Anderson RCB calculator [25]. RCB could not be calculated in patients who underwent ALND before neoadjuvant chemotherapy. We categorized patients into two groups: favorable RCB (RCB-0, RCB-I) and unfavorable RCB (RCB-II, RCB-III).

\section{Statistical analysis}

All analyses were performed using PASW Statistics for Windows version 18.0 (SPSS, Inc., Chicago, IL, USA). T-, chi-square, and Fisher's exact tests were used to compare clinicopathologic factors between the soft and hard, pCR and non-pCR, and favorable RCB and unfavorable RCB groups. Univariate and multivariate logistic regression analysis were performed to evaluate the correlations between clinicopathological factors and pCR or favorable RCB. Multivariate logistic regression analysis using backward elimination was performed with significant variables ( $p<0.10$ in univariate analysis). Statistical significance was set at $p<0.05$.

\section{RESULTS}

\section{Clinicopathologic characteristics of patients}

The mean patient age and initial tumor size were $46.43 \pm 8.62$ years and $3.63 \pm 1.95 \mathrm{~cm}$, respectively. Strain elastography was performed in 74 patients, $24(32.4 \%)$ and $50(67.6 \%)$ of whom were categorized into the soft and hard groups, respectively. In six patients, pathologic factors, except hormone receptor (HR), HER2 and Ki-67, could not be evaluated from CNB specimens because of their poor condition. Histologic grade, TILs, cellularity and characteristics of stroma were analyzed from $89 \mathrm{CNB}$ specimens (Table 1).

\section{Comparison of clinicopathologic factors between soft} and hard groups according to strain elastography

No significant differences in HR and HER2 positivity or nuclear grade 3 were observed between the soft and hard groups. The mean tumor cellularity from $\mathrm{CNB}$ specimens was $43.86 \pm 21.04 \%$ in the soft group and $39.18 \pm 14.41 \%$ in the hard group $(p=0.35)$. Stromal characteristics did not differ between the two groups $(p=0.79)$. The rates of pCR or favorable RCB did not differ significantly between the two groups ( $p=0.28$, and $p=0.30$, respectively) (Table 2 ).

\section{Comparisons of clinicopathologic factors between pCR and non-pCR groups}

Twenty-two patients (23.2\%) achieved a pCR. The clinical stage did 
Table 1. Clinicopathologic characteristics of the patients $(n=95)$

\begin{tabular}{|c|c|}
\hline Characteristic & $\begin{array}{l}\text { Value } \\
\text { No. (\%) }\end{array}$ \\
\hline Age (yr) & $46.43 \pm 8.62$ (range, $27-71)$ \\
\hline \multicolumn{2}{|l|}{ Menopausal status } \\
\hline Premenopause & $63(66.3)$ \\
\hline Postmenopause & $32(33.7)$ \\
\hline Initial tumor size on US (cm) & $3.63 \pm 1.95$ (range, $2.1-12.28$ ) \\
\hline \multicolumn{2}{|l|}{ Clinical T stage } \\
\hline $\mathrm{T} 2$ & $85(89.5)$ \\
\hline T3 & $4(4.2)$ \\
\hline T4 & $6(6.3)$ \\
\hline \multicolumn{2}{|l|}{ Clinical nodal metastasis } \\
\hline Positive & $55(57.9)$ \\
\hline Negative & $40(42.1)$ \\
\hline \multicolumn{2}{|l|}{ Clinical stage } \\
\hline ॥ & $73(76.8)$ \\
\hline III & $22(23.2)$ \\
\hline \multicolumn{2}{|l|}{ Tumor elasticity on elastography* } \\
\hline Soft (Tsukuba score 1, 2, 3) & $24(32.4)$ \\
\hline Hard (Tsukuba score 4, 5) & $50(67.5)$ \\
\hline \multicolumn{2}{|l|}{ Axillary operation before chemotherapy } \\
\hline Yes & $45(47.4)$ \\
\hline No & $50(52.6)$ \\
\hline \multicolumn{2}{|l|}{ Histologic type } \\
\hline Invasive carcinoma, no special type & $73(76.8)$ \\
\hline Invasive lobular carcinoma & $2(2.1)$ \\
\hline Mixed type or miscellaneous & $6(6.3)$ \\
\hline Not available & $14(14.7)$ \\
\hline \multicolumn{2}{|l|}{ ER } \\
\hline Positive & $53(55.8)$ \\
\hline Negative & $42(44.2)$ \\
\hline \multicolumn{2}{|l|}{ PR } \\
\hline Positive & $43(45.3)$ \\
\hline Negative & $52(54.7)$ \\
\hline \multicolumn{2}{|l|}{ HER2 } \\
\hline Positive & $34(35.8)$ \\
\hline Negative & $61(64.2)$ \\
\hline \multicolumn{2}{|l|}{ Nuclear grade on $\mathrm{CNB}^{\dagger}$} \\
\hline 1 & $1(1.1)$ \\
\hline 2 & $18(20.2)$ \\
\hline 3 & $70(78.7)$ \\
\hline \multicolumn{2}{|l|}{ Ki-67 index on CNB (\%) } \\
\hline$\leq 20$ & $17(17.9)$ \\
\hline$>20$ & $78(82.1)$ \\
\hline Mean TILs level on CNB ${ }^{\dagger}(\%)$ & $9.36 \pm 13.43$ (range, $0-50$ ) \\
\hline \multicolumn{2}{|l|}{ TILs on CNB ${ }^{\dagger}(\%)$} \\
\hline$<10$ & $56(62.9)$ \\
\hline$\geq 10$ & $33(37.1)$ \\
\hline
\end{tabular}

(Continued to the next)
Table 1. Continued

\begin{tabular}{lc}
\hline Characteristic & $\begin{array}{c}\text { Value } \\
\text { No. (\%) }\end{array}$ \\
\hline Tumor cellularity on $\mathrm{CNB}^{\dagger}(\%)$ & $42.08 \pm 16.60$ (range, 5-90) \\
Characteristics of stroma ${ }^{\dagger}$ & \\
Fibroblastic & $29(32.6)$ \\
Collagenous & $38(42.7)$ \\
Myxoid & $22(24.7)$ \\
Reduction rate of tumor size between & $16.83 \pm 12.30$ \\
1st and 2nd US $(\%)^{\ddagger}$ & (range, $-15.00-42.31)$ \\
\hline
\end{tabular}

Values are presented as mean \pm standard deviation or number (\%).

$\mathrm{US}=$ ultrasonography; $\mathrm{ER}=$ estrogen receptor; $\mathrm{PR}=$ progesterone receptor; HER2 = human epidermal growth factor receptor 2; CNB = core needle biopsy; TILs = tumor infiltrating lymphocytes. ${ }^{*} \mathrm{n}=74 ;{ }^{+} \mathrm{n}=89 ;{ }^{*} \mathrm{n}=47$.

not differ between the $\mathrm{pCR}$ and non-pCR groups. Tumor elasticity was not correlated with $\mathrm{pCR}(p=0.28)$. Patients with a $\mathrm{pCR}$ were more likely to have ER- or PR-negative breast cancer compared to those without a $\operatorname{pCR}$ ( $p=0.04$ and $p=0.03$, respectively). The rate of nuclear grade 3 was higher in patients with $\mathrm{pCR}$ than in those without $\mathrm{PCR}$ $(95.5 \%$ vs. $73.1 \%, p=0.03)$. There also was no significant difference in tumor cellularity $(p=0.34)$ or stromal characteristics $(p=0.95)$ between the two groups (Table 3).

\section{Comparisons of clinicopathologic factors between favorable and unfavorable RCB groups}

RCB was calculated for 79 patients. Thirty patients (38.0\%) achieved favorable RCB, while 49 patients (62.0\%) had unfavorable RCB. There was no significant difference in clinical stage and tumor elasticity between the two groups. The proportion of patients with ER negativity was higher in the favorable RCB group than that in the unfavorable $\mathrm{RCB}$ group; however, the difference showed borderline significance ( $63.3 \%$ vs. $40.8 \%, p=0.05)$. Compared to the unfavorable RCB group (49\%), the favorable RCB group was significantly more likely to have PR-negative breast cancer $(73.3 \%, p=0.03)$. Nuclear grade 3 was observed in $96.4 \%$ of patients in the favorable RCB group and $73.3 \%$ in the unfavorable RCB group $(p=0.01)$. The rate of patients with $\geq 10 \%$ TILs was higher in the favorable RCB group than those in the unfavorable RCB group $(p=0.04)$. No significant differences were observed in the other pathologic factors between the two groups (Table 3). 
Table 2. Comparisons of clinicopathologic factors between soft and hard groups according to strain elastography

\begin{tabular}{|c|c|c|c|}
\hline \multirow[b]{2}{*}{ Clinicopathologic factor } & \multicolumn{2}{|c|}{ Strain elastography } & \multirow[b]{2}{*}{$p$-value } \\
\hline & $\begin{array}{c}\text { Soft group }(n=24) \\
\text { No. }(\%)\end{array}$ & $\begin{array}{c}\text { Hard group }(n=50) \\
\text { No. }(\%)\end{array}$ & \\
\hline Age (yr) & $45.67 \pm 9.35$ & $47.42 \pm 7.91$ & 0.40 \\
\hline \multicolumn{4}{|l|}{ Menopausal status } \\
\hline Premenopause & $16(66.7)$ & $32(64.0)$ & \multirow[t]{2}{*}{0.82} \\
\hline Postmenopause & $8(33.3)$ & $18(36.0)$ & \\
\hline Initial tumor size on US (cm) & $3.56 \pm 2.32$ & $3.23 \pm 1.10$ & 0.40 \\
\hline \multicolumn{4}{|l|}{ Clinical T stage } \\
\hline $\mathrm{T} 2$ & $21(87.5)$ & $47(94.0)$ & \multirow[t]{3}{*}{0.19} \\
\hline $\mathrm{T} 3$ & 0 & $2(4.0)$ & \\
\hline T4 & $3(12.5)$ & $1(2.0)$ & \\
\hline \multicolumn{4}{|l|}{ Clinical nodal metastasis } \\
\hline Positive & $12(50.0)$ & $27(54.0)$ & \multirow[t]{2}{*}{0.75} \\
\hline Negative & $12(50.0)$ & $23(46.0)$ & \\
\hline \multicolumn{4}{|l|}{ Clinical stage } \\
\hline ॥ & $16(66.7)$ & $44(88.0)$ & \multirow[t]{2}{*}{0.03} \\
\hline III & $8(33.3)$ & $6(12.0)$ & \\
\hline \multicolumn{4}{|l|}{ ER } \\
\hline Positive & $14(58.3)$ & $30(60.0)$ & \multirow[t]{2}{*}{0.89} \\
\hline Negative & $10(41.7)$ & $20(40.0)$ & \\
\hline \multicolumn{4}{|l|}{$\mathrm{PR}$} \\
\hline Positive & $11(45.8)$ & $24(48.0)$ & \multirow[t]{2}{*}{0.86} \\
\hline Negative & $13(54.2)$ & $26(52.0)$ & \\
\hline \multicolumn{4}{|l|}{ HER2 } \\
\hline Positive & $8(33.3)$ & $20(40.0)$ & \multirow[t]{2}{*}{0.58} \\
\hline Negative & $16(66.7)$ & $30(60.0)$ & \\
\hline \multicolumn{4}{|l|}{ Nuclear grade on CNB* } \\
\hline 1 or 2 & $3(13.6)$ & $15(30.6)$ & \multirow[t]{2}{*}{0.15} \\
\hline 3 & $19(86.4)$ & $34(69.4)$ & \\
\hline \multicolumn{4}{|l|}{ Ki-67 index on CNB (\%) } \\
\hline$\leq 20$ & $3(12.5)$ & $13(26.0)$ & \multirow[t]{2}{*}{0.24} \\
\hline$>20$ & $21(87.5)$ & $37(74.0)$ & \\
\hline Mean TILs level on CNB* (\%) & $13.68 \pm 16.64$ & $6.78 \pm 10.60$ & 0.08 \\
\hline \multicolumn{4}{|l|}{ TILs on CNB* $(\%)$} \\
\hline$<10$ & $11(50.0)$ & $34(69.4)$ & 0.12 \\
\hline$\geq 10$ & $11(50.0)$ & $15(30.6)$ & \\
\hline Tumor cellularity on CNB* (\%) & $43.86 \pm 21.04$ & $39.18 \pm 14.41$ & 0.35 \\
\hline Characteristics of stroma* & & & \\
\hline Fibroblastic & $6(27.3)$ & $16(32.7)$ & 0.79 \\
\hline Collagenous & $10(45.5)$ & $23(46.9)$ & \\
\hline Myxoid & $6(27.3)$ & $10(20.4)$ & \\
\hline $\begin{array}{l}\text { Reduction rate of tumor size between 1st and } \\
2 \text { nd US }^{+}(\%)\end{array}$ & $20.51 \pm 15.51$ & $17.81 \pm 10.89$ & 0.54 \\
\hline Breast operation methods & & & \\
\hline $\mathrm{BCS}$ & $20(83.3)$ & $46(92.0)$ & 0.26 \\
\hline Mastectomy or SCM & $4(16.7)$ & $4(8.0)$ & \\
\hline Pathologic response to neoadjuvant chemother & & & \\
\hline $\mathrm{pCR}$ & $7(29.2)$ & $9(18.0)$ & 0.28 \\
\hline Non-pCR & $17(70.8)$ & $41(82.0)$ & \\
\hline Residual cancer Burden ${ }^{\ddagger}$ & & & \\
\hline Favorable RCB & $10(47.6)$ & $14(34.1)$ & 0.30 \\
\hline Unfavorable RCB & $11(52.4)$ & $27(65.9)$ & \\
\hline
\end{tabular}

Values are presented as mean \pm standard deviation or number (\%).

$\mathrm{US}=$ ultrasonography; $\mathrm{ER}=$ estrogen receptor; $\mathrm{PR}=$ progesterone receptor; $\mathrm{HER2}=$ human epidermal growth factor receptor 2; $\mathrm{CNB}=$ core needle biopsy; $\mathrm{TIL} s=$ tumor infiltrating lymphocytes; $\mathrm{BCS}=$ breast conserving surgery; $\mathrm{SCM}=$ subcutaneous mastectomy; $\mathrm{PCR}=$ pathologic complete response; $\mathrm{RCB}=$ residual cancer burden.

${ }^{*} \mathrm{n}=71 ;{ }^{\dagger} \mathrm{n}=40 ;{ }^{*} \mathrm{n}=62$. 
Table 3. Comparison of clinicopathologic factors between the $\mathrm{pCR}$ and non-pCR groups, and favorable RCB and unfavorable RCB groups

\begin{tabular}{|c|c|c|c|c|c|c|}
\hline \multirow[b]{2}{*}{ Clinicopathologic factor } & \multicolumn{3}{|c|}{ pCR group vs. non-pCR group } & \multicolumn{3}{|c|}{ Favorable RCB vs. unfavorable RCB } \\
\hline & $\begin{array}{c}\text { pCR group } \\
\text { No. }(\%)(n=22)\end{array}$ & $\begin{array}{l}\text { Non-pCR group } \\
\text { No. }(\%)(n=73)\end{array}$ & $p$-value & $\begin{array}{c}\text { Favorable RCB group } \\
\text { No. }(\%)(n=30)\end{array}$ & $\begin{array}{c}\text { Unfavorable RCB } \\
\text { No. }(\%) \text { group }(n=49)\end{array}$ & $p$-value \\
\hline Age (yr) & $47.09 \pm 9.73$ & $46.23 \pm 8.32$ & 0.69 & $47.03 \pm 9.16$ & $45.84 \pm 8.77$ & 0.57 \\
\hline \multicolumn{7}{|l|}{ Menopausal status } \\
\hline Premenopause & $14(63.6)$ & $49(67.1)$ & 0.76 & $20(66.7)$ & $31(63.3)$ & 0.76 \\
\hline Postmenopause & $8(36.4)$ & $24(32.9)$ & & $10(33.3)$ & $18(36.7)$ & \\
\hline Initial tumor size on US (cm) & $3.81 \pm 2.12$ & $3.57 \pm 1.90$ & 0.61 & $3.52 \pm 1.89$ & $3.68 \pm 2.20$ & 0.74 \\
\hline \multicolumn{7}{|l|}{ Clinical T stage } \\
\hline $\mathrm{T} 2$ & $20(91.0)$ & $65(89.0)$ & 1 & $28(93.3)$ & $44(89.8)$ & 1 \\
\hline T3 & $1(4.5)$ & $3(4.1)$ & & $1(3.3)$ & $2(4.1)$ & \\
\hline T4 & $1(4.5)$ & $5(6.9)$ & & $1(3.3)$ & $3(6.1)$ & \\
\hline \multicolumn{7}{|l|}{ Clinical nodal metastasis } \\
\hline Positive & $12(54.5)$ & $33(58.9)$ & 0.72 & $14(46.7)$ & $25(51.0)$ & 0.71 \\
\hline Negative & $10(45.5)$ & $40(41.1)$ & & $16(53.3)$ & $24(49.0)$ & \\
\hline \multicolumn{7}{|l|}{ Clinical stage } \\
\hline II & $15(68.2)$ & $58(79.5)$ & 0.27 & $23(76.7)$ & $40(81.6)$ & 0.59 \\
\hline III & $7(31.8)$ & $15(20.5)$ & & $7(23.3)$ & $9(18.4)$ & \\
\hline \multicolumn{7}{|l|}{ Tumor elasticity on elastography* } \\
\hline Soft (Tsukuba score 1, 2, 3) & $7(43.8)$ & $17(29.3)$ & 0.28 & $10(41.7)$ & $11(28.9)$ & 0.30 \\
\hline Hard (Tsukuba score 4, 5) & $9(56.2)$ & $41(70.7)$ & & $14(58.3)$ & $27(71.1)$ & \\
\hline \multicolumn{7}{|l|}{ ER } \\
\hline Positive & $8(36.4)$ & $45(61.6)$ & 0.04 & $11(36.7)$ & $29(59.2)$ & 0.05 \\
\hline Negative & $14(63.6)$ & $28(38.4)$ & & $19(63.3)$ & $20(40.8)$ & \\
\hline \multicolumn{7}{|l|}{$\mathrm{PR}$} \\
\hline Positive & $5(22.7)$ & $38(52.1)$ & 0.03 & $8(26.7)$ & $25(51.0)$ & 0.03 \\
\hline Negative & $17(77.3)$ & $35(47.9)$ & & $22(73.3)$ & $24(49.0)$ & \\
\hline \multicolumn{7}{|l|}{ HER2 } \\
\hline Positive & $11(50.0)$ & $23(31.5)$ & 0.13 & $14(46.7)$ & $14(28.6)$ & 0.10 \\
\hline Negative & $11(50.0)$ & $50(68.5)$ & & $16(53.3)$ & $35(71.4)$ & \\
\hline \multicolumn{7}{|l|}{ Nuclear grade on $\mathrm{CNB}^{\dagger}$} \\
\hline 1 or 2 & $1(4.5)$ & $18(26.9)$ & 0.03 & $1(3.6)$ & $12(26.7)$ & 0.01 \\
\hline 3 & $21(95.5)$ & $49(73.1)$ & & $27(96.4)$ & $33(73.3)$ & \\
\hline \multicolumn{7}{|l|}{ Ki-67 index on CNB (\%) } \\
\hline$\leq 20$ & $2(9.1)$ & $15(20.5)$ & 0.22 & $3(10.0)$ & $10(20.4)$ & 0.35 \\
\hline$>20$ & $20(90.9)$ & $58(79.5)$ & & $27(90.0)$ & $39(79.6)$ & \\
\hline Mean TILs level on CNB ${ }^{\dagger}(\%)$ & $13.09 \pm 15.72$ & $8.13 \pm 12.47$ & 0.13 & $14.07 \pm 15.45$ & $7.07 \pm 12.15$ & 0.05 \\
\hline \multicolumn{7}{|l|}{ TILs on CNB ${ }^{\dagger}(\%)$} \\
\hline$<10$ & $11(50.0)$ & $45(67.2)$ & 0.18 & $13(46.4)$ & $32(71.1)$ & 0.04 \\
\hline$\geq 10$ & $11(50.0)$ & $22(32.8)$ & & $15(53.6)$ & $13(28.9)$ & \\
\hline Tumor cellularity on $\mathrm{CNB}^{\dagger}(\%)$ & $45.00 \pm 18.71$ & $41.12 \pm 15.88$ & 0.34 & $42.86 \pm 17.61$ & $43.78 \pm 15.27$ & 0.82 \\
\hline \multicolumn{7}{|l|}{ Characteristics of stroma $^{+}$} \\
\hline Fibroblastic & $7(31.8)$ & $22(32.8)$ & 0.95 & $10(35.7)$ & $16(35.6)$ & 0.95 \\
\hline Collagenous & $9(40.9)$ & $29(43.3)$ & & $12(42.9)$ & $18(40.0)$ & \\
\hline Myxoid & $6(27.3)$ & $16(23.9)$ & & $6(21.6)$ & $11(24.4)$ & \\
\hline $\begin{array}{l}\text { Reduction rate of tumor size } \\
\left.\text { between } 1 \text { st and } 2 \text { nd US } \text { US }^{\ddagger} \%\right)\end{array}$ & $20.83 \pm 13.92$ & $15.46 \pm 11.59$ & 0.20 & $22.37 \pm 12.72$ & $13.44 \pm 11.5$ & 0.03 \\
\hline \multicolumn{7}{|l|}{ Breast operation methods } \\
\hline BCS & $20(90.9)$ & $60(82.2)$ & 0.33 & $28(93.3)$ & $38(77.6)$ & 0.12 \\
\hline Mastectomy or SCM & $2(9.1)$ & $13(17.8)$ & & $2(6.7)$ & $11(22.4)$ & \\
\hline
\end{tabular}

Values are presented as mean \pm standard deviation or number (\%).

$\mathrm{pCR}=$ pathologic complete response; $\mathrm{RCB}=$ residual cancer burden; $\mathrm{US}=$ ultrasonography; $\mathrm{ER}=$ estrogen receptor; $\mathrm{PR}=$ progesterone receptor; $\mathrm{HER} 2=$ human epidermal growth factor receptor 2; $\mathrm{CNB}=$ core needle biopsy; TILs= tumor infiltrating lymphocytes; $\mathrm{BCS}=$ breast conserving surgery; $\mathrm{SCM}=$ subcutaneous mastectomy.

${ }^{*} n=74$ (according to $p C R$ ), 62 (according to $R C B$ ); ${ }^{\dagger} n=89$ (according to $p C R$ ), 73 (according to $R C B$ ); ${ }^{\ddagger} n=47$ (according to $p C R$ ), 41 (according to RCB). 
Table 4. Univariate and multivariate analysis of clinicopathologic factors associated with $\mathrm{pCR}$ or favorable RCB

\begin{tabular}{|c|c|c|c|c|c|c|c|c|c|c|c|c|}
\hline \multirow{3}{*}{ Clinicopathologic factor } & \multicolumn{6}{|c|}{$\mathrm{pCR}$} & \multicolumn{6}{|c|}{ Favorable RCB } \\
\hline & \multicolumn{3}{|c|}{ Univariate anaylsis } & \multicolumn{3}{|c|}{ Multivariate anaylsis } & \multicolumn{3}{|c|}{ Univariate anaylsis } & \multicolumn{3}{|c|}{ Multivariate anaylsis } \\
\hline & OR & $95 \% \mathrm{Cl}$ & $p$-value & $\mathrm{OR}$ & $95 \% \mathrm{Cl}$ & $p$-value & $\mathrm{OR}$ & $95 \% \mathrm{Cl}$ & $p$-value & OR & $95 \% \mathrm{Cl}$ & $p$-value \\
\hline Initial tumor size & 1.06 & $0.85-1.33$ & 0.61 & - & - & - & 0.96 & $0.77-1.21$ & 0.74 & - & - & - \\
\hline $\begin{array}{l}\text { Clinical LN metastasis } \\
\text { positive vs. negative }\end{array}$ & 0.84 & $0.32-2.19$ & 0.72 & - & - & - & 0.84 & $0.34-2.09$ & 0.71 & - & - & - \\
\hline $\begin{array}{l}\text { Tumor elasticity } \\
\text { hard vs. soft }\end{array}$ & 0.53 & $0.17-1.66$ & 0.28 & - & - & - & 0.57 & $0.20-1.67$ & 0.31 & - & - & - \\
\hline $\begin{array}{l}\text { Hormone receptor } \\
\text { negative vs. positive }\end{array}$ & 2.81 & $1.05-7.56$ & 0.04 & 2.44 & $0.87-6.85$ & 0.09 & 2.51 & $0.98-6.39$ & 0.06 & 2.93 & $1.04-8.28$ & 0.04 \\
\hline $\begin{array}{l}\text { HER2 } \\
\text { positive vs. negative }\end{array}$ & 2.17 & $0.82-5.74$ & 0.12 & - & - & - & 2.19 & $0.85-5.65$ & 0.11 & - & - & - \\
\hline $\begin{array}{l}\text { Nuclear grade } \\
3 \text { vs. } 1,2\end{array}$ & 7.71 & $0.97-61.60$ & 0.05 & 5.77 & $0.70-47.67$ & 0.10 & 9.82 & $1.20-80.37$ & 0.03 & 6.79 & $0.79-58.02$ & 0.08 \\
\hline $\begin{array}{r}\text { Ki-67 index (\%) } \\
>20 \text { vs. } \leq 20\end{array}$ & 2.59 & $0.54-12.31$ & 0.23 & - & - & - & 2.31 & $0.58-9.18$ & 0.24 & - & - & - \\
\hline $\begin{array}{l}\text { TILs (\%) } \\
\quad \geq 10 \text { vs. }<10\end{array}$ & 2.05 & $0.77-5.45$ & 0.15 & - & - & - & 2.84 & $1.06-7.59$ & 0.04 & NS & & \\
\hline
\end{tabular}

$\mathrm{pCR}=$ pathologic complete response; $\mathrm{RCB}=$ residual cancer burden; $\mathrm{OR}=$ odds ratio; $\mathrm{Cl}=$ confidence interval; $\mathrm{LN}=$ lymph node; $\mathrm{HER} 2=$ human epidermal growth factor 2; TlLs= tumor infiltrating lymphocytes; NS= not significant.

Univariate and multivariate analysis for

clinicopathologic factors associated with $\mathrm{pCR}$ or favorable RCB

Univariate analysis showed a significant association between HR negativity $(p=0.04)$ and $\mathrm{pCR}$ and a borderline significant association between nuclear grade $3(p=0.05)$ and pCR (Table 4$)$. In multivariate analysis, no factors showed a significant correlation with pCR, although the odds ratio (OR) of nuclear grade 3 was 5.77 (95\% confidence interval $[\mathrm{CI}], 0.70-47.67 ; p=0.10)$ and that of HR negativity was $2.44[95 \% \mathrm{CI}, 0.87-6.85 ; p=0.09)$.

Univariate analysis showed a significant correlation between high TIL level $(\geq 10 \%)(p=0.04)$, nuclear grade $3(p=0.03)$, and favorable RCB (Table 4). In multivariate analysis, HR negativity was an independent predictor of favorable RCB (OR, 2.93; 95\% CI, 1.04-8.28; $p=0.04)$.

\section{DISCUSSION}

Neoadjuvant chemotherapy can facilitate BCS in locally advanced breast cancer and convert inoperable cases into possible ones [10]. pCR is associated with a good prognosis $[2,3]$. However, not all patients achieved favorable results after neoadjuvant chemotherapy.
Thus, it is important to select appropriate patients with clinicopathologic factors that are expected to respond well to neoadjuvant chemotherapy.

Recently, many articles have reported on elastography as a potential tool for predicting the response to neoadjuvant chemotherapy [19-21]. Hayashi et al. [19] evaluated tumor elasticity by strain elastography using the Tsukuba elasticity scoring system and found that tumor elasticity was an independent predictor of $\mathrm{pCR}$ in multivariate analysis. Ma et al. [21] reported strain elastography and shear wave elastography as predictive tools for a favorable pathologic response to neoadjuvant chemotherapy for breast cancer.

In contrast to previous studies [19-21], tumor elasticity was not a predictive factor for response to neoadjuvant chemotherapy in our study. Strain elastography (as used in the present study) is performed by light manual compression of the probe above the targeted lesion, whereas shear wave elastography was performed using acoustic radiation force from a transducer. Thus, the sensitivity of strain elastography is lower than that of shear wave elastography. Chang et al. [26] reported that tumor location, size, and depth can influence elastography quality. Uneven breast surfaces caused by irregularly-shaped tumors can also lead to uneven manual compression. Recent studies have reported that the change in tumor elasticity induced by neoadjuvant 
chemotherapy is a significant predictive factor for the response to neoadjuvant chemotherapy $[16,20]$. The necrotic area of the tumor due to neoadjuvant chemotherapy usually appears as a soft lesion on elastography. Ma et al. [21] reported that changes in strain or shear wave elastography after two cycles of neoadjuvant chemotherapy play an important predictive role. In this study, we evaluated the elasticity of the tumor only before neoadjuvant chemotherapy. Although we failed to prove that elastography predicted the pathologic responses, serial changes in tumor elasticity on pathologic responses may predict tumor response to treatment. The stromal characteristics in $\mathrm{CNB}$ specimens were not correlated with tumor elasticity in the current study, whereas those from surgically resected specimens were correlated with tumor elasticity in a study by Chamming et al. [16]. CNB specimens may not be representative of whole tumor, especially a large breast cancer. Furthermore, we categorized the stromal characteristics according to the dominant component of the stroma only. The percentages of each stromal component in the mixed stromal types were not considered. Elastography with US is a noninvasive imaging modality for assessing the response of the whole tumor. This may be help to predict the response and determine the optimal time for surgery during neoadjuvant chemotherapy. Further studies with more cases are needed to evaluate tumor elasticity as a potential tool for predicting the response to neoadjuvant chemotherapy.

In our study, HR negativity was an independent predictive factor of favorable RCB. Regarding pCR, HR negativity was a significant predictive factor in univariate analysis, although not in multivariate analysis. Other studies have also reported breast cancer subtypes as predictive tools for neoadjuvant chemotherapy [4-7]. Moon et al. [27] reported a higher pCR rate in HR-negative patients. Boughey et al. [4] and Gentile et al. [5] reported a significantly higher pCR rate in triple-negative and HER2-positive breast cancers compared to that in HR-positive breast cancer. Rouzier et al. [6] also reported significantly different rates of pCR among molecular subtypes.

Histologic grade is reportedly correlated with neoadjuvant chemotherapy response. Miyoshi et al. [28] found that nuclear grade 3 was also a significant predictor of non-pCR. However, the univariate analysis in the present study showed a significant association between nuclear grade 3 and favorable RCB and a borderline significant association between nuclear grade 3 and pCR. Most articles reported results similar to ours. Jaegal et al. [29] reported a higher pCR in patients with a high histologic grade. Wang et al. [30] and Shien et al. [7] reported significantly higher rates of $\mathrm{pCR}$ in patients with nuclear grade $3 \mathrm{com}-$ pared to those in patients with nuclear grades 1 or 2. Additionally, nuclear grade 3 was a significant predictive factor for pCR in a study by Osako et al. [31]. Jung et al. [9] also reported that histologic grade 3 was an independent predictor of pCR in Korean patients with triple-negative breast cancer.

Several studies have reported TILs as predictors of pCR. Jung et al. [9] reported that high TIL levels were significantly associated with pCR in triple-negative breast cancer. Denkert et al. [8] reported intratumoral lymphocytes as an independent predictor of pCR. Yamaguchi et al. [10] found that dense lymphocytic infiltration was a significant independent predictive factor for pCR. In the present study, a significantly higher percentage of patients with $\geq 10 \%$ TIL showed favorable RCB compared to those with unfavorable RCB, although $\geq 10 \%$ TIL was not significantly correlated with favorable RCB in multivariate analysis.

Our study had several limitations. First, we included a small number of cases from a single center. Second, strain elastography could not be performed in 21 patients. In addition, 47 cases underwent a second US after the first cycle of neoadjuvant chemotherapy, whereas 33 cases, underwent follow-up studies by PET. The heterogeneity of imaging tools for follow-up after the first cycle of neoadjuvant chemotherapy made it difficult to interpret the reduction rate of tumor size between the first and second US.

Tumor elasticity has recently emerged as a predictive factor for neoadjuvant chemotherapy response. In this study, tumor elasticity was not associated with pCR or RCB. HR was an independent predictor of RCB. Further large prospective studies are needed to determine the correlation between tumor elasticity or other clinicopathological factors and neoadjuvant chemotherapy response.

\section{CONFLICT OF INTEREST}

The authors declare that they have no competing interests.

\section{REFERENCES}

1. Clinical practice guidelines in oncology, version 2. 2017. National Comprehensive Cancer Network. https://www.nccn.org/profes- 
sionals/physician_gls/pdf/breast.pdf. Accessed Apr 6th, 2017.

2. von Minckwitz G, Untch M, Blohmer JU, Costa SD, Eidtmann H, Fasching PA, et al. Definition and impact of pathologic complete response on prognosis after neoadjuvant chemotherapy in various intrinsic breast cancer subtypes. J Clin Oncol 2012;30:1796-804.

3. Cortazar P, Zhang L, Untch M, Mehta K, Costantino JP, Wolmark N, et al. Pathological complete response and long-term clinical benefit in breast cancer: the CTNeoBC pooled analysis. Lancet 2014;384: 164-72.

4. Boughey JC, McCall LM, Ballman KV, Mittendorf EA, Ahrendt GM, Wilke LG, et al. Tumor biology correlates with rates of breast-conserving surgery and pathologic complete response after neoadjuvant chemotherapy for breast cancer: findings from the ACOSOG Z1071 (alliance) prospective multicenter clinical trial. Ann Surg 2014;260:608-14.

5. Gentile LF, Plitas G, Zabor EC, Stempel M, Morrow M, Barrio AV. Tumor biology predicts pathologic complete response to neoadjuvant chemotherapy in patients presenting with locally advanced breast cancer. Ann Surg Oncol 2017;24:3896-902.

6. Rouzier R, Perou CM, Symmans WF, Ibrahim N, Cristofanilli M, Anderson K, et al. Breast cancer molecular subtypes respond differently to preoperative chemotherapy. Clin Cancer Res 2005;11:567885.

7. Shien T, Akashi-Tanaka S, Miyakawa K, Hojo T, Shimizu C, Seki K, et al. Clinicopathological features of tumors as predictors of the efficacy of primary neoadjuvant chemotherapy for operable breast cancer. World J Surg 2009;33:44-51.

8. Denkert C, Loibl S, Noske A, Roller M, Muller BM, Komor M, et al. Tumor-associated lymphocytes as an independent predictor of response to neoadjuvant chemotherapy in breast cancer. J Clin Oncol 2010;28:105-13.

9. Jung YY, Hyun CL, Jin MS, Park IA, Chung YR, Shim B, et al. Histomorphological factors predicting the response to neoadjuvant chemotherapy in triple-negative breast cancer. J Breast Cancer 2016; 19:261-7.

10. Yamaguchi R, Tanaka M, Yano A, Tse GM, Yamaguchi M, Koura K, et al. Tumor-infiltrating lymphocytes are important pathologic predictors for neoadjuvant chemotherapy in patients with breast cancer. Hum Pathol 2012;43:1688-94.

11. Fasching PA, Heusinger K, Haeberle L, Niklos M, Hein A, Bayer
CM, et al. Ki67, chemotherapy response, and prognosis in breast cancer patients receiving neoadjuvant treatment. BMC Cancer 2011;11:1-13.

12. MacGrogan G, Mauriac L, Durand M, Bonichon F, Trojani M, de Mascarel I, et al. Primary chemotherapy in breast invasive carcinoma: predictive value of the immunohistochemical detection of hormonal receptors, p53, c-erbB-2, MiB1, pS2 and GST pi. Br J Cancer 1996;74:1458-65.

13. MacGrogan G, Rudolph P, Mascarel Id I, Mauriac L, Durand M, Avril A, et al. DNA topoisomerase II alpha expression and the response to primary chemotherapy in breast cancer. Br J Cancer 2003; 89:666-71

14. Buchholz TA, Garg AK, Chakravarti N, Aggarwal BB, Esteva FJ, Kuerer HM, et al. The nuclear transcription factor kappaB/bcl-2 pathway correlates with pathologic complete response to doxorubicin-based neoadjuvant chemotherapy in human breast cancer. Clin Cancer Res 2005;11:8398-402.

15. Choi IK, Strauss R, Richter M, Yun CO, Lieber A. Strategies to increase drug penetration in solid tumors. Front Oncol 2013;3:193.

16. Chamming's F, Latorre-Ossa H, Le Frere-Belda MA, Fitoussi V, Quibel T, Assayag F, et al. Shear wave elastography of tumour growth in a human breast cancer model with pathological correlation. Eur Radiol 2013;23:2079-86.

17. Lee JH, Kim SH, Kang BJ, Choi JJ, Jeong SH, Yim HW, et al. Role and clinical usefulness of elastography in small breast masses. Acad Radiol 2011;18:74-80

18. Sadigh G, Carlos RC, Neal CH, Dwamena BA. Ultrasonographic differentiation of malignant from benign breast lesions: a meta-analytic comparison of elasticity and BIRADS scoring. Breast Cancer Res Treat 2012;133:23-35.

19. Hayashi M, Yamamoto Y, Ibusuki M, Fujiwara S, Yamamoto S, Tomita S, et al. Evaluation of tumor stiffness by elastography is predictive for pathologic complete response to neoadjuvant chemotherapy in patients with breast cancer. Ann Surg Oncol 2012;19: 3042-9.

20. Jing H, Cheng W, Li ZY, Ying L, Wang QC, Wu T, et al. Early evaluation of relative changes in tumor stiffness by shear wave elastography predicts the response to neoadjuvant chemotherapy in patients with breast cancer. J Ultrasound Med 2016;35:1619-27.

21. Ma Y, Zhang S, Li J, Li J, Kang Y, Ren W. Comparison of strain and 
shear-wave ultrasounic elastography in predicting the pathological response to neoadjuvant chemotherapy in breast cancers. Eur Radiol 2017;27:2282-91.

22. Itoh A, Ueno E, Tohno E, Kamma H, Takahashi H, Shiina T, et al. Breast disease: clinical application of US elastography for diagnosis. Radiology 2006;239:341-50.

23. Salgado R, Denkert C, Demaria S, Sirtaine N, Klauschen F, Pruneri $\mathrm{G}$, et al. The evaluation of tumor-infiltrating lymphocytes (TILs) in breast cancer: recommendations by an international TILs working group 2014. Ann Oncol 2015;26:259-71.

24. Symmans WF, Peintinger F, Hatzis C, Rajan R, Kuerer H, Valero V, et al. Measurement of residual breast cancer burden to predict survival after neoadjuvant chemotherapy. J Clin Oncol 2007;25:441422.

25. Residual cancer burden calculator. The university of Texas MD Anderson cancer center. http://www3.mdanderson.org/app/medcalc/ index.cfm?pagename=jsconvert3. Accessed May 6th, 2018.

26. Chang JM, Moon WK, Cho N, Kim SJ. Breast mass evaluation: factors influencing the quality of US elastography. Radiology 2011;259: $59-64$.
27. Moon T, Cho DH, Youn JM, Lee JB, Bae JW, Jung SP. Evaluation of prognostic factors and validation of tumor response ratios after neoadjuvant chemotherapy in patients with breast cancer. J Breast Dis 2016;4:108-15.

28. Miyoshi Y, Kurosumi M, Kurebayashi J, Matsuura N, Takahashi M, Tokunaga E, et al. Low nuclear grade but not cell proliferation predictive of pathological complete response to docetaxel in human breast cancers. J Cancer Res Clin Oncol 2008;134:561-7.

29. Jaegal M, Kang SJ, Ryu YJ, Cho JS, Park MH, Yoon JH. Prognostic factors in patients with locally advanced breast cancer treated by neoadjuvant chemotherapy. J Breast Dis 2019;7:23-9.

30. Wang J, Buchholz TA, Middleton LP, Allred DC, Tucker SL, Kuerer $\mathrm{HM}$, et al. Assessment of histologic features and expression of biomarkers in predicting pathologic response to anthracycline-based neoadjuvant chemotherapy in patients with breast carcinoma. Cancer 2002;94:3107-14.

31. Osako T, Horii R, Matsuura M, Domoto K, Ide Y, Miyagi Y, et al. High-grade breast cancers include both highly sensitive and highly resistant subsets to cytotoxic chemotherapy. J Cancer Res Clin Oncol 2010;136:1431-8. 